\title{
Synthesis and Transformation of Carbonaceous Nanoparticles
}

\author{
Vito Mennella \\ INAF - Osservatorio Astronomico di Capodimonte, via Moiariello, 1680131 Napoli, Italy \\ email: mennella@na.astro.it
}

\begin{abstract}
The physical properties of carbonaceous nanoparticles depend on the production conditions. In addition, these properties are modified by heat, UV and ion irradiation and gas interaction. We will discuss the synthesis and transformation of carbon nanoparticles that have been proposed as carriers of aromatic and aliphatic spectroscopic features observed in the interstellar medium.
\end{abstract}

\section{Carbon particles in space}

The presence in the diffuse interstellar medium of aromatic and aliphatic carbon components is inferred from the UV interstellar extinction bump and the $3.4 \mu \mathrm{m}$ absorption band, respectively. Interstellar and circumstellar objects in our Galaxy and many extragalactic objects emit a spectrum characterized by features generally attributed to aromatic carbon molecules such as polycyclic aromatic hydrocarbon molecules (PAHs). Fullerene have recently been confirmed in many sources of different nature. The variety of carbon species present in space is due to the nature of the carbon atom. It can have three bonding configurations $s p^{3}, s p^{2}$ and $s p$ that give rise to allotropic forms (diamond, graphite, fullerene and carbine or carbonoid material) with different optical and physical properties. Besides these forms there is great variety of disordered materials that are characterized by mixtures of carbon bonding configurations. Their properties depend on the prevailing bonding configuration. In carbon materials the atomic organization is closely related to the electronic structure and, consequently, to the optical properties.

\section{Carbon particles in the laboratory}

To get insight into the nature of the interstellar carbon components, analogue materials have been studied. Different methods, such as arc discharge, laser ablation, chemical vapor deposition, flame synthesis and energetic processing of carbon containing molecules at low temperature, have been applied for the production of carbon nanoparticles. The produced samples have been studied with different analytic techniques to obtain information on structure and optical properties. Laboratory simulation of grain processing in space is very important to interpret the changes of grains in different interstellar environments through an evolutionary physical-chemical scheme of materials. In fact, dust grain composition evolves through exposure to UV photons, heat, gas and cosmic rays.

On the basis of the experimental results of UV irradiation of hydrogenated carbon grains a model for the UV interstellar extinction bump was proposed (Mennella et al. 1998). The model relies on the extrapolation of the laboratory trend of the dielectric function as function of the UV dose to represent the UV processing pathway for hydrogenated carbon grains in the diffuse medium. Recent experimental work confirms that the UV extinction bump is consistent with UV irradiated hydrogenated carbon grains (Gadallah et al. 2011). 
Energetic processing by UV photons and ions of aliphatic materials destroys their $\mathrm{C}-\mathrm{H}$ bonds. UV irradiation of hydrogenated carbon grains and hydrocarbon molecules under simulated dense and diffuse medium conditions is indeed characterized by a strong decrease of the $3.4 \mu \mathrm{m}$ band (Mennella et al. 2001, Muñoz Caro et al. 2001, Gadallah et al. 2012). On the basis of the experimental results one can conclude that the interstellar radiation field should destroy the $\mathrm{C}-\mathrm{H}$ bonds aliphatic component of diffuse regions in a very short time scale $\left(10^{4}\right.$ years $)$. A similar destruction of the aliphatic $\mathrm{C}-\mathrm{H}$ bonds is observed during ion irradiation of hydrogenated carbon grains under simulated dense and diffuse interstellar medium conditions (Mennella et al. 2003, Godard et al. 2011).

Simulation of carbon dust processing under diffuse and dense medium conditions indicates that the key process for the evolution of the interstellar aliphatic carbon component is its interaction with $\mathrm{H}$ atoms. This interaction is able to counteract the destruction of $\mathrm{C}-\mathrm{H}$ bonds by UV photons and cosmic rays and it activates the $3.4 \mu \mathrm{m}$ band in diffuse interstellar regions and the $3.47 \mu \mathrm{m}$ feature in dense clouds (Mennella et al. 2002, Mennella 2008). The interesting aspect is that the same carbon grain population can absorb at the two wavelengths as a consequence of evolutionary transformations caused by processing. The transformations are compatible with the time-scale required by fast cycling of materials between dense and diffuse regions of the interstellar medium.

Moreover, this carbon component, after the inclusion in a comet during the formation process in the cold outer edge of the solar nebula, may evolve to develop the $\mathrm{CH}_{2}$ and $\mathrm{CH}_{3}$ groups (as suggested by laboratory simulations) and contribute to the aliphatic band at $3.4 \mu \mathrm{m}$ of Interplanetary Dust Particles and particles of comet Wild 2 collected by the Stardust mission (Mennella 2010).

\section{Acknowledgements}

This work has been supported by ASI research contracts.

\section{References}

Gadallah, K. A. K., Mutschke, H., \& Jager, C. 2011, A\& A 528, A56

Gadallah, K. A. K., Mutschke, H., \& Jager, C. 2012, A\&A 544, A107

Godard, M., et al. 2011, A\& A 529, 146

Mennella, V. 2008, ApJ 682, L101

Mennella, V. 2010, ApJ 718, 867

Mennella, V., Colangeli, L., Bussoletti, E., Palumbo, P., \& Rotundi, A. 1998, ApJ 507, L177

Mennella, V., Muñoz Caro, G., Ruiterkamp, R., Schutte, W. A., Greenberg, J. M., Brucato, J. R., \& Colangeli, L. 2001, A\& A 367, 355

Mennella, V., Brucato, J. R., Colangeli, L., \& Palumbo, P. 2002, ApJ 569, 531

Mennella, V., Baratta, G. A., Esposito, A., Ferini, G., \& Pendleton, Y. J. 2003, ApJ 587, 727

Muñoz Caro, G., Ruiterkamp, R., Schutte, W. A., Greenberg, J. M., \& Mennella, V. 2001, A\&A 367,347 